\title{
Molecular Gas in the Central Regions of Barred Galaxies
}

\author{
Jeffrey Kenney \\ Yale University, Astronomy Department, P.O. Box 208101, New Haven, \\ CT 06520-8101 USA
}

\begin{abstract}
The morphology and kinematics of molecular gas in the central regions of barred galaxies are described. The largest gas concentrations are often located near ILRs, although there is a range of morphologies. The gas motions associated with star-forming rings are predominantly circular, while motions just beyond the rings are often non-circular and in some cases show clear radial inflow. In barred galaxies with circumnuclear starbursts in early phases of evolution, the CO is centrally peaked, perhaps inside IILRs. The most intense star formation occurs where the gas motions are circular, and where the rotation curve rises steeply and is nearly solid body.
\end{abstract}

\section{Introduction}

Many spiral galaxies contain a large concentration of molecular gas, located deep within a bar and often coincident with the central part of a stellar bulge. These circumnuclear gas disks are often kinematically distinct from surrounding gas, associated with inner Lindblad resonances (ILRs), and comprise a significant fraction of the dynamical mass (Kenney et al. 1992; Turner 1994). It is here in the central few hundred pc where the bar-driven radial inflow of gas slows, and much of the gas forms stars, sometimes in a starburst. The stars formed in these compact gaseous disks build circumnuclear stellar disks and perhaps stellar bulges. In this paper I describe the morphology and kinematics of molecular gas in the central regions of barred galaxies, based principally on high resolution $\left(\sim 2^{\prime \prime}\right)$ CO observations with the Owens Valley Interferometer. A more extensive discussion of these and related topics is contained in a review by Kenney (1995).

\section{Circumnuclear Rings and ILRs}

The great variety in circumnuclear gas morphologies is illustrated by the OVRO CO maps in Figure 1 for seven galaxies with bars of at least moderate strength. There are rings or partial rings (NGC 4314), spiral arms (NGC 6951), "twin peaks" (NGC 3351), filled exponential disks (NGC 3504, NGC 4102), or some combination of these. Sc galaxies, particularly those with weaker stellar bars, have strong $\mathrm{CO}$ emission along the linear or slightly curved ridges which lead into the circumnuclear region (M101, M83), while the Sa (NGC 4314) and Sb (NGC 3351, NGC 3504, NGC 4102, NGC 6951) galaxies tend to have weak 

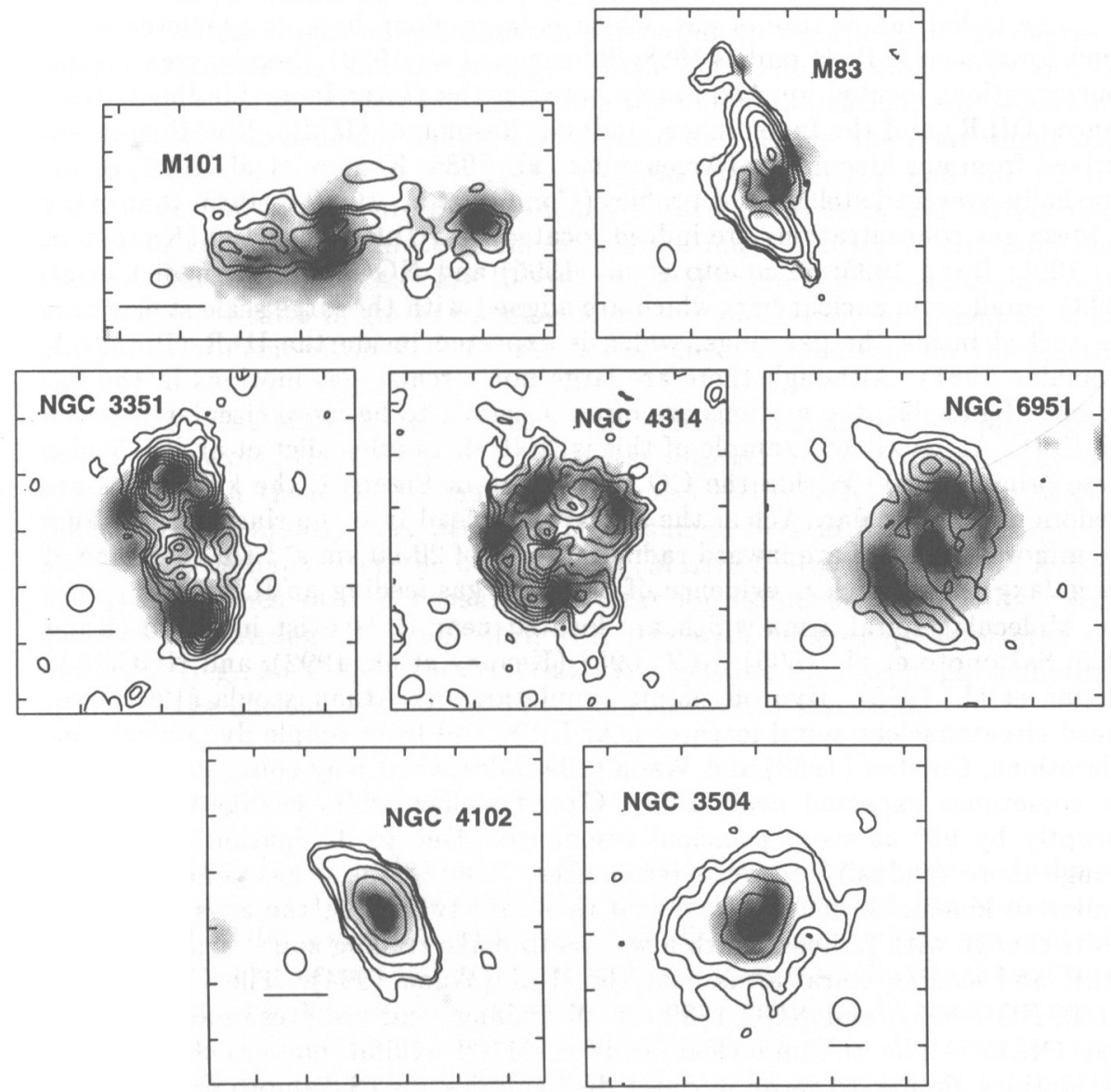

Figure 1. $\quad \mathrm{CO}$ contour and $\mathrm{H} \alpha+[\mathrm{N} \mathrm{II}]$ greyscale maps of the circumnuclear regions of seven galaxies, illustrating the variety of circumnuclear gas morphologies. All galaxies have large-scale stellar bars which extend beyond the areas shown in these maps. The strongest $\mathrm{CO}$ emission is located close to ILRs in NGC 3351, NGC 6951, NGC 4314, and perhaps also in M101 and M83. In the young starbursts NGC 3504 and NGC 4102 the gas is centrally concentrated, inside OILRs and perhaps inside IILRs. NGC 3351, NGC 6951, M101, and NGC 3504 from Kenney et al. (1992), NGC 4314 from Benedict et al. (1995), M83 from Turner et al. (1995), NGC 4102 from Jogee \& Kenney (1995). Beamsizes, which are typically $2-3^{\prime \prime}$ (except $7^{\prime \prime} \times 4^{\prime \prime}$ for M83), are shown as ellipses. Vertical tickmarks are separated by $5^{\prime \prime}$ (except $15^{\prime \prime}$ for M83). The horizontal line in each panel is $300 \mathrm{pc}$ in length. 
$\mathrm{CO}$ emission along the bar. Although the variety in Figure 1 is only partially understood, the largest gas concentrations are often located near ILRs.

The radial inflow rate of gas, which is large along bars, is predicted to be much lower near ILRs (Combes 1988; Shlosman et al. 1989), thereby creating gas concentrations located approximately between the Outer Inner Lindblad Resonance (OILR) and the Inner Inner Lindblad Resonance (IILR). Rotation curves derived from gas kinematics (Arsenault et al. 1988; Kenney et al. 1992) or azimuthally averaged stellar light profiles (Combes et al. 1992) confirm that many of these gas concentrations are indeed located near ILRs. In M100 (Knapen et al. 1995; Rand 1995; Sakamoto et al. 1995) and NGC 4314 (Benedict et al. 1993), small scale nuclear bars which are aligned with the large scale stellar bars are tucked inside the gas rings, which is expected inside the IILR (Binney \& Tremaine 1987). Although there are large non-circular gas motions in the bar regions of galaxies, the motions are often observed to be more circular between the ILRs. An excellent example of this is NGC 4314 (Benedict et al. 1995; also these proceedings). Within the $\mathrm{CO}$ ring shown in Figure 1, the kinematics are predominantly circular. Yet in the spurs which lead into the ring and lie along the minor axis, there are inward radial motions of $20-90 \mathrm{~km} \mathrm{~s}^{-1}$ (in the plane of the galaxy). This is clear evidence of inflowing gas feeding an ILR ring.

Molecular spiral arms which are located near ILRs exist in M100 (Rand 1995; Sakamoto et al. 1995), NGC 6951 (Kenney et al. 1992), and NGC 1530 (Regan et al. 1995). Hydrodynamic simulations by Athanassoula (1992) produced circumnuclear spiral features near ILRs, and from simple dynamical considerations, Combes (1988) and Wada (1994) described why spiral gas patterns are sometimes expected near ILRs. Closed stellar orbits change orientation abruptly by $90^{\circ}$ at every principal resonance. Due to dissipation, gas orbits change more gradually between resonances, forming spiral gas concentrations similar to kinematic density waves. If there are two ILRs, the axes of the gas orbits change with radius in such a way as to make trailing spiral arms near the OILR and leading spiral arms near the IILR (Wada 1994). The CO arms in M100, NGC 6951, and NGC 1530 are all trailing, and are presumably located near OILRs. The circumnuclear arms in M100 exhibit non-circular streaming motions similar to those in outer disk spiral arms (Sakamoto et al. 1995). Leading gaseous arms near the IILR have not yet been observed, although nearinfrared images of M100 suggest modest leading arms in the stellar distribution in the vicinity of the putative IILR (Knapen et al. 1995; also these proceedings).

In NGC 3351, the strongest CO emission arises from "twin peaks", located symmetrically about the nucleus, and oriented perpendicular to the large-scale bar (Kenney et al. 1992). These peaks are where inward flowing gas from the bar, traced by faint $\mathrm{CO}$ emission coincident with the dust lanes along the leading edge of the stellar bar, appears to intersect a ring of gas on more circular orbits at the ILR. The twin peaks are probably regions of orbit crowding where two kinematically distinct gas streams meet, similar to bar ends (Kenney \& Lord 1991). This phenomenon appears distinct from spiral arm formation. Although the twin peaks are particularly prominent in NGC 3351, similar features can be recognized in Figure 1 in M101 and NGC 6951. The region of strongest CO emission in the northern CO spiral arm of NGC 6951 is where the CO spiral arm intersects a partial ring of $\mathrm{CO}$ and $\mathrm{H} \alpha$ emission. $\mathrm{CO}$ spectra near this northern 
peak show broad (FWHM $\geq 100 \mathrm{~km} \mathrm{~s}^{-1}$ ), complex line profiles with two or more peaks, perhaps indicating the kinematics of two interacting gas streams.

The variety in gas morphologies is partly due to variations in the degree of central concentration and the strength of the bar. The rotation curve and the pattern speed of the stellar bar determine the number, location, and separation of the ILRs. Gas behavior should also depend on whether there are small scale nuclear bars (Shlosman et al. 1989; Wozniak et al. 1995), and the relative PAs of the bars if they are independently rotating. Some of the variety must be due to evolutionary effects. Timescales are much shorter in circumnuclear regions: starburst timescales are $10^{7}-10^{8}$ yrs, and dynamical timescales are $\sim 10^{7}$ yrs. While a wealth of gas morphologies can be understood in principal, it has not yet been demonstrated which factors are most responsible for the existing variety.

What is the ultimate fate of gas which is driven radially inward along bars? In many galaxies, the regions of intense star formation are more centrally concentrated than the CO distributions (note especially NGC 6951 and M83 in Figure 1). Apparently very little gas is consumed by star formation along the bar (except perhaps when the bar is young (Martin \& Roy 1995; Friedli \& Benz 1995)), and much of it is consumed by star formation near the ILRs. Some galaxies have strong $\mathrm{CO}$ inside their ILR ring with little or no associated star formation (e.g., NGC 3351 and NGC 4314), indicating that gas might sometimes approach the nucleus without being consumed by star formation.

\section{Starbursts}

Barred galaxies in the early stages of the most intense circumnuclear starbursts have their gas centrally concentrated. Figure 1 shows that the young starbursts NGC 3504 and NGC 4102 have CO distributions which are centrally peaked and nearly azimuthally symmetric, in contrast with the five other ("nonstarbursting") galaxies in this Figure, which have significantly lower circumnuclear luminosities and lower ratios of luminosity to gas mass. In these two starburst galaxies the $\mathrm{CO}$ radial distributions out to $\sim 1 \mathrm{kpc}$ are nearly exponential with scale lengths of $\sim 300$ pc (Kenney et al. 1993; Jogee \& Kenney 1995; also these proceedings), an order of magnitude more compact than the exponential molecular gas disks which inhabit the outer disks of many Sc galaxies.

In these young starbursts, most of the gas lies within the region where the gas has nearly circular orbits. The gas depletion timescale, inferred from rate of star formation per unit gas mass, is shortest in the center, where the rotation curve rises steeply and is nearly solid body (Kenney et al. 1993). Consequently, these starbursts will evolve from the inside out. The gas surface density (assuming the 'standard' $\mathrm{CO}-\mathrm{H}_{2}$ value) is approximately equal to the Toomre critical surface density in the starburst region of NGC 3504 (Kenney et al. 1993), so the simple gravitational instability theory of star formation seems to apply in circumnuclear starbursts as well as outer disks (Kennicutt 1989).

Are these young starbursts dynamically different from the "non-starbursts" which have rings of gas and star formation between ILRs? Both the rotation curve and the dust lane morphology of NGC 3504 indicate the presence of ILRs. The region containing most of the gas and star formation is inside the OILR, 
although it is not clear whether it lies between the OILR and IILR or extends inside the IILR (Kenney et al. 1993). It may be that the largest gas concentrations and the most intense star formation are generally confined to the annulus between ILRs (Arsenault et al. 1988; Kenney et al. 1992; Knapen et al. 1995), and the region between the ILRs is wide in some galaxies. Alternatively, perhaps the most intense starbursts occur only when large quantities of gas get inside the IILR.

The behavior of gas inside the ILR is poorly understood, yet is critical for understanding starbursts. Although gravitational torques are the dominant radial driver of gas along large-scale stellar bars (Combes 1988), other effects might become dominant inside the ILR. If rapidly rotating, small-scale nuclear bars form inside the ILR, gas may be driven further inward by gravitational torques (Shlosman et al. 1989). Gas might collapse rapidly inside the IILR once the gas mass is sufficiently large to become self-gravitating (Wada \& Habe 1992; Heller \& Shlosman 1994). Gas may drift toward the center as a consequence of dynamical friction on Giant Molecular Clouds (Stark et al. 1991; Combes et al. 1992) or viscous torques (Lesch et al. 1990). Alternatively, perhaps large quantities of gas are driven deeply toward the nucleus only when there is a large dynamical disturbance, such as a tidal interaction or merger, and orbits are so perturbed and transient that there is no meaningful ILR.

The starbursts in NGC 3504 and NGC 4102 appear to be in very early stages of evolution. Their $\mathrm{CO}$ distributions are not ringlike despite the fact that gas is being consumed more rapidly in the center (Kenney et al. 1993; Jogee \& Kenney 1995). NGC 4102 has a compact ionized gas outflow with expansion timescale of only $10^{6} \mathrm{yr}$ (Boer 1994). In these galaxies, the starburst has not yet consumed or blown out much of the central gas. Since starbursts consume their gas most rapidly in the center, and also generate outflowing winds, they are predicted to have ring-like gas distributions at later times. The well-studied, nearby, edge-on galaxies M82 and NGC 253 are evolved starbursts, containing freely expanding winds with outflow timescales of $3-10 \times 10^{6} \mathrm{yr}$ (Heckman et al. 1990). Some of the "non-starbursts" in Figure 1 with rings of gas and star formation may be in a post-starburst phase. However, since it is also possible to produce gas rings from dynamical effects, an understanding of both dynamical and evolutionary effects is essential for understanding circumnuclear regions.

Circumnuclear gas disks spawn circumnuclear starbursts which build compact circumnuclear stellar disks, and perhaps even stellar bulges. The central few hundred $p c$ of many galaxies contain compact circumnuclear stellar disks, as revealed by spiral structure and rotational velocities which are large compared to their velocity dispersion (Kormendy 1993). Due to their compactness, some of these disks were previously thought to be the dense central portions of bulges. The starburst in NGC 3504 is in the process of making or adding to one of these compact stellar disks. Its central CO radial distribution is exponential with a scale length of $300 \mathrm{pc}$, similar to the radial distribution of $K$-band light (Kenney et al. 1993). The high gas surface density and relatively low CO velocity dispersion $\left(2000 \mathrm{M}_{\odot} \mathrm{pc}^{-2}\right.$ and $20 \mathrm{~km} \mathrm{~s}^{-1}$ at $\left.\mathrm{r}=200 \mathrm{pc}\right)$ imply the progenitor gas disk is thin. Theory and simulations show that stars in an originally thin disk may be driven to large scale heights via scattering at the vertical resonances of a bar (Pfenniger \& Norman 1990; Friedli \& Benz 1995). This mechanism operating on young circumnuclear stellar disks may contribute to bulge building. 


\section{References}

Arsenault, R., Boulesteix, J., Georgelin, Y., \& Roy, J.-R. 1988, A\&A200, 29

Athanassoula, E. 1992, MNRAS, 259, 345

Benedict, G. F., et al. 1993, AJ, 105, 1369

Benedict, G. F., Smith, B., \& Kenney, J. D. P. 1995, in preparation

Binney, J. \& Tremaine, S. 1987, Galactic Dynamics, Princeton: Princeton University Press

Boer, B. 1994, in Mass-Transfer Induced Activity in Galaxies, I. Shlosman, Cambridge: Cambridge University Press, 308

Combes, F. 1988, in Galactic and Extragalactic Star Formation R. E. Pudritz $\&$ M. Fich, Dordrecht: Kluwer, 475

Combes, F., Gerin, M., Nakai, N., Kawabe, R., \& Shaw, M. A. 1992, A\&A, 259, $\mathrm{L} 27$

Friedli, D. \& Benz, W. 1995, A\&A, 301, 649

Heckman, T. M., Armus, L. \& Miley, G. K. 1990, ApJS, 74, 833

Heller, C. H. \& Shlosman, I. 1994, ApJ, 424, 84

Jogee, S. \& Kenney, J. D. P. 1995, in preparation

Kenney, J. D. P. 1995, in The Interstellar Medium in Galaxies, J. M. van der Hulst, Dordrecht: Kluwer, in press

Kenney, J. D. P., Carlstrom, J. E., \& Young, J. S. 1993, ApJ, 418, 687

Kenney, J. D. P. \& Lord, S. D. 1991, ApJ, 381, 118

Kenney, J. D. P., Wilson, C. D., Scoville, N. Z., Devereux, N. A., \& Young, J. S. 1992, АpJ, 395, L79

Kennicutt, R. C., Jr. 1989, ApJ, 344, 685

Knapen, J. H., Beckman, J. E., Shlosman, I., Peletier, R. F., Heller, C. H., \& de Jong, R. S. 1995, ApJ, 443, L73

Kormendy, J. 1993, in Galactic Bulges, H. Dejonghe \& H. J. Habing, Dordrecht: Kluwer, 209

Lesch, H., Biermann, P. L., Crusius, A., Reuter, H. P., Dahlem, M., Barteldrees, A., \& Wielebinski, R. 1990, MNRAS, 242, 194

Martin, P. \& Roy, J.-R. 1995, ApJ, 445, 161

Pfenniger, D. \& Norman, C. 1990, ApJ, 363, 391

Rand, R. J. 1995, AJ, 109, 2444

Regan, M. W., Vogel, S. N., \& Teuben, P. 1995, ApJ, in press

Sakamoto, K., Okumura, S., Minezaki, T., Kobayashi, Y., \& Wada, K. 1995, AJ, 110,2075

Shlosman, I., Frank, J., \& Begelman, M. C. 1989, Nature, 338, 45

Stark, A. A., Gerhard, O. E., Binney, J., \& Bally, J. 1991, MNRAS, 248, 14P

Turner, J. L. 1994, in Mass-Transfer Induced Activity in Galaxies, I. Shlosman, Cambridge: Cambridge University Press, 90

Turner, J. L., Hurt, R. L., \& Kenney, J. D. P. 1995, in preparation

Wada, K. \& Habe, A. 1992, MNRAS, 258, 82 
Wada, K. 1994, PASJ, 46, 165

Wozniak, H., Friedli, D., Martinet, L., Martin, P., \& Bratschi, P. 1995 A\&AS, 111,115

\section{Discussion}

B. Elmegreen: You expect that there will be regions in the inner parts of galaxies where much of the gas will be in diffuse molecular ( $\mathrm{CO}$ ) form. This results from the high pressure in these regions and from the relative ease of self-shielding for $\mathrm{H}_{2}$ at high pressure. Some of the $\mathrm{CO}$ peaks without star formation in your maps could be due to diffuse molecular clouds.

$J$. Kenney: Even if it is diffuse, there may be a lot of gas mass there. A recent study by Magnani \& Onello (1995) found that the $\mathrm{CO}-\mathrm{H}_{2}$ ratio varied significantly among diffuse Milky Way molecular clouds, but the mean value was close to the "standard" value for gravitationally-bound clouds.

B. Elmegreen: Do any of these galaxies with strong $\mathrm{CO}$ nuclear emission have AGN's? If not, do you think the gas is turning into stars?

$J$. Kenney: NGC 6951 has a Seyfert nucleus, but very little CO emission at the nucleus. It could be like NGC 1068, which has weak CO but a very high $\mathrm{HCN} / \mathrm{CO}$ ratio near the nucleus, indicating that virtually all the gas is very dense, probably due to energy input from the AGN. The starbursts NGC 3504 and NGC 4102 are LINERs, and may have AGN in addition to star formation.

B. Koribalski: How does the gas get inside the ILR? Could it be that a nuclear spiral (in contrast to a nuclear ring) between the OILR and IILR helps to drive the gas inside?

J. Kenney: The torques from a nuclear spiral could drive gas radially between the OILR and IILR, but may not be strong enough to drive gas inside the IILR.

Z. Tsvetanov: Do you have a feeling whether the gas fraction increases toward the nucleus, and if yes, what sort of gradient do you see.

$J$. Kenney: This varies among galaxies. For the galaxies in Figure 1 with a gas ring, the enclosed gas mass fraction peaks on the ring. For the centrally peaked starbursts, the enclosed gas mass fraction is centrally peaked. In NGC 3504, it is $40 \%$ at $100 \mathrm{pc}$, and falls to $20 \%$ at $500 \mathrm{pc}$.

J. A. Garcia-Barreto: Do the CO isovelocity contours in the central region of NGC 4314 show any evidence for the mini-bar seen in the HST images?

$J$. Kenney: There are small deviations $\left(\leq 10 \mathrm{~km} \mathrm{~s}^{-1}\right)$ from circular motion near the nuclear bar, in a pattern mostly consistent with bar streaming motions, although this is just above the noise level. These non-circular motions are much smaller than those just outside the ring.

$J$. Turner: What effect does the self-gravity of the gas have in view of the high molecular gas mass fraction?

$J$. Kenney: The gas self-gravity may help drive inflow and may also affect the formation and transmission of density waves (e.g., passing through ILRs). 